\title{
Avaliação da Prevalência do Diabetes e da Hiperglicemia de Estresse no Infarto Agudo do Miocárdio
}

\begin{abstract}
RESUMO
Objetivos: Determinar a prevalência do diabetes melito (DM) e da hiperglicemia de estresse (HE) em pacientes com infarto agudo do miocárdio (IAM) admitidos em unidade de emergência cardiológica. Métodos: Análise retrospectiva de 2.262 pacientes com IAM, avaliando, além da prevalência de diabetes referido, o diagnosticado e a hiperglicemia de estresse. Resultados: Apesar de referido em 12,1\% dos pacientes (H: 10,7\%, M: 15,8\%), o DM ocorria efetivamente em 24,8\% (H: 22,9\%, M: 29,7\%) e a HE em 13,6\% (H: 14,3\%, $\mathrm{M}: 11,7 \%)$ dos indivíduos dessa população. Portanto, alterações glicêmicas ocorreram em 37,4\% dos indivíduos com IAM (H: 37,2\%, M: 41,4\%). Nos pacientes com DM, observou-se maior precocidade etária do IAM, maior prevalência de óbitos (DM: 20,7\%, ND:13,8\%, HE: 13,4\%) e de procedimentos cirúrgicos (ND: 33,8\%, HE: 18,0\%, DM: 21,7\%). Conclusão: A elevada prevalência de DM e hiperglicemia de estresse observada em nosso estudo indica que as alterações glicêmicas constituem um dos mais importantes fatores de risco para o IAM. (Arq Bras Endocrinol Metab 2008; 52/3:465-472)
\end{abstract}

Descritores: Diabetes; Complicações crônicas do diabetes; Diabetes e doença cardiovascular; Infarto do miocárdio

\section{ABSTRACT}

The Prevalence of Diabetes and Stress Hyperglycemia in the Acute Myocardial Infarction Patients.

Objectives: To evaluate in our population the real prevalence of diabetes (DM) and stress hyperglycemia (HE) in patients with myocardial infarction (IAM) admitted in a cardiologic emergency unit. Methods: A retrospective analysis of 2262 patients with AMI evaluating the prevalence of DM (referred and diagnosed) and stress hyperglycemia. Results: Besides $12,1 \%$ of subjects were previously referred to be diabetic (men: $10.7 \%$ and women: $15.8 \%$ ), diabetes was effectively diagnosed in 24,8\% (M: 22,9\%, W: 29,7\%) and stress hyperglycemia in $13,6 \%$ HE of the patients (M: $14,3 \%, \mathrm{~W}: 11,7 \%$ ) indicating that glycemic alterations were effectively observed in $37.2 . \%$ of the patients with IAM (M: $37,2 \%, W: 41,4 \%$ ). In DM subjects IAM events occurred earlier, total intra-hospital mortality was higher (DM: 20.7\%, ND: 13,8\%, HE: 13,4\%) and less surgical procedures were performed (ND 33.8\%, DM: 21.7\%, HE: 18.0\%). Conclusion: The elevated DM and stress hyperglycemia prevalence observed in our study indicates that glycemic alterations is one of the most important risk factors for IAM. (Arq Bras Endocrinol Metab 2008; 52/3:465-472)

Keywords: Complications of diabetes; Diabetes and cardiovascular heart disease; Myocardial infarction

\section{artigo original}

\author{
antonio Carlos lerario \\ Fabiana M. L. Martins CorettI \\ Sergio FerReira de Oliveira \\ Roberto Tadeu Barcelos BettI \\ MARIA DO SOCORRO C. B. BASTOS \\ LetícIa de Araujo Funari FerrI \\ Rosa Maria RaHMI GarcIa \\ BERNARDO LEO WAJCHENBERG
}

Núcleo de Diabetes e Coração do Instituto do Coração (Incor) da Faculdade de Medicina da Universidade de São Paulo (FM-USP), SP, Brasil (ACL, FMLMC, SFO, RTBB, LAFF, RMRG, BLW); Disciplina de Endocrinologia do Hospital das Clínicas da FMUSP, SP, Brasil (ACL, MSCBB, BLW)

Recebido em 02/01/2007 Aceito em 05/10/2007 


\section{INTRODUÇÃO}

DIABETES TIPO 2 É UMA doença muito freqüente, e sua prevalência está aumentando em praticamente todo o mundo, estimando-se que o número de indivíduos diabéticos deverá sofrer ainda um incremento de $50 \%$ até o ano de 2025 (1-3). Por estar associado ao desenvolvimento de diversas complicações crônicas, especialmente as doenças cardiovasculares, esse recente aumento explosivo vem ganhando crescente importância como fator de desenvolvimento de eventos cardiovasculares agudos (4-6). O infarto do miocárdio (IAM) e o acidente vascular cerebral (AVC) constituem atualmente as principais causas de morte dos pacientes com diabetes, e o diabetes é descrito como importante causa de morte secundária de doenças circulatórias (4,7-9). Estima-se que o risco de um paciente diabético desenvolver a doença coronariana é duas a três vezes maior do que um indivíduo não-diabético (10) e que apesar de ter sido descrita redução da mortalidade geral em eventos agudos coronarianos, estes índices de mortalidade referentes ao diabetes estão se elevando (11). A associação entre o diabetes e a doença cardiovascular tem sido demonstrada não somente nos pacientes que apresentam a forma clínica da doença, definida por glicemias de jejum superiores a $126 \mathrm{mg} / \mathrm{dL}$ ou glicemias casuais superiores a $200 \mathrm{mg} / \mathrm{dL}$, mas também em fases mais precoces e assintomáticas da história natural da doença, cujas glicemias de jejum estão alteradas (entre $100 \mathrm{mg} /$ dL e $126 \mathrm{mg} / \mathrm{dL}$ ) ou apresentam elevação das glicemias pós-prandiais (entre $140 \mathrm{mg} / \mathrm{dL}$ e $200 \mathrm{mg} / \mathrm{dL}$ ), denominadas respectivamente de pré-diabetes (ou disglicemia) e intolerância à glicose (12-14). A relevância dessas alterações glicêmicas precoces como fator de risco para o desenvolvimento da doença cardiovascular tem sido bem evidenciada por diversos estudos clínicos, especialmente o realizado em 117 mil enfermeiras norte-americanas avaliadas prospectivamente por 20 anos, que observou incidência significativamente maior de doenças cardiovasculares no grupo de mulheres que já apresentavam ou desenvolveram o diabetes e a intolerância à glicose em um período que chegava a preceder a forma clínica do diabetes em até 15 anos (15).

A presença de hiperglicemia no período de admissão de indivíduos com IAM que não referiam diabetes é geralmente conhecida como "hiperglicemia do estresse" por ser atribuída ao estímulo adrenérgico resultante do estresse associado ao evento isquêmico miocárdico. Sua importância clínica tem sido reconhecida não somente por indicar uma condição de menor reserva pancreática e conseqüentemente por alterações do metabolismo glicêmico, mas também por estar associada a piores desfechos imediatos e tardios do IAM quando comparados a pacientes não-diabéticos (16-23).

Entretanto, apesar de seu elevado impacto na gênese e sua gravidade do IAM, a importância do diabetes e especialmente das alterações glicêmicas mais precoces e menos intensas pode ser muitas vezes subestimada, uma vez que, o diabetes é geralmente referido como causa secundária de mortalidade e ou de internações hospitalares em virtude de doenças circulatórias (9). São poucos ou limitados a pequenas coortes de pacientes, especialmente em nosso meio (24), os estudos epidemiológicos que indiquem especificamente os índices da prevalência do diabetes e da hiperglicemia de estresse nos eventos agudos coronarianos. Portanto, no presente estudo visamos a mais bem determinar a prevalência do diabetes e da hiperglicemia de estresse em pacientes com IAM e analisar sua relação com a mortalidade e o tipo de estratégia terapêutica empregada no período de admissão hospitalar.

\section{CASUÍSTICA E MÉTODOS}

Foram analisados, em estudo retrospectivo, prontuários de atendimento de 2.756 pacientes atendidos com o diagnóstico de IAM nos anos de 1997, 1998 e 1999 na unidade de emergência do Instituto do Coração do Hospital das Clínicas da Faculdade de Medicina da Universidade de São Paulo (InCor). Como vários pacientes apresentaram nesse período mais do que um atendimento, incluímos no presente estudo somente os 2.446 prontuários de primeira consulta, quando ocorreu o episódio de IAM. Posteriormente, excluímos do estudo 174 indivíduos por não apresentarem registros de glicemias nos prontuários durante o período de internação e seis pacientes por apresentarem idades inferiores a 30 anos de idade. Portanto, foram incluídos no estudo 2.262 pacientes (1.622 homens e 640 mulheres). Consideramos como critério de diagnóstico do diabetes: 1) relato de diabetes feito por parte do paciente ou por acompanhantes na história clínica de entrada; e 2) indivíduos que apresentaram duas ou mais glicemias casuais iguais ou superiores a $200 \mathrm{mg} / \mathrm{dL}$, um dos critérios definidos pela Organização Mundial da Saúde (OMS) para o diagnóstico do diabetes (25) que utilizamos por não podermos precisar perfeitamente o período de jejum 
dos pacientes. Consideramos como hiperglicemia de estresse a presença de duas ou mais glicemias com valores entre $140 \mathrm{mg} / \mathrm{dL}$ e $199 \mathrm{mg} / \mathrm{dL}$. Foram também avaliadas as relações da presença de diabetes e da hiperglicemia em função das faixas etárias dos pacientes, do sexo, do desfecho e do tipo de tratamento (clínico ou cirúrgico) realizado durante o período de internação. Todas as determinações glicêmicas foram realizadas no laboratório de patologia clínica do InCor, utilizando método enzimático em analisador automatizado, sendo os valores obtidos dos laudos emitidos pelo laboratório.

Para a análise e a estatística realizada utilizou-se o software SPSS 10.0 for Windows. As prevalências apresentadas foram expressadas em médias e em valores percentuais. A análise estatística para avaliar a associação entre as faixas etárias divididas em décadas, entre os tipos de tratamento e dos desfechos foram feitos com base no teste do qui-quadrado.

\section{RESULTADOS}

A prevalência do diabetes e a intolerância à glicose dos pacientes estudados são apresentadas na tabela 1 . Dos 2.262 pacientes com IAM avaliados, observou-se a pre- sença do diabetes em 274 indivíduos (12,11\%) com base na informação referida na história clínica de entrada ao hospital e em 287 indivíduos (12,69\%) após a análise das glicemias durante o período de internação, que, quando somados, elevam a $561(24,80 \%)$ o número de pacientes efetivamente diagnosticados como diabéticos. A análise individualizada para cada sexo mostrou prevalência relativa de diabetes (referido + observado) mais elevada de diabetes nas mulheres $(29,69 \%)$ do que nos homens $(22,87 \%)(\mathrm{p}<0,05)$. A avaliação das glicemias durante a internação evidenciou também que 307 indivíduos $(13,57 \%$ do total estudado) apresentaram hiperglicemia de estresse, dos quais 232 eram do sexo masculino $(14,30 \%$ do total de homens) e 75 do sexo feminino (11,72\% do total de mulheres). Quando agrupamos os indivíduos que apresentaram diabetes (referido e diagnosticado) e com hiperglicemia de estresse constatamos que $868(38,37 \%)$ dos 2.262 pacientes com IAM apresentavam alteração da homeostase glicemia, cujos valores percentuais se mostraram mais elevados nas mulheres $(41,41 \%)$ do que nos homens $(37,18 \%)(\mathrm{p}<0,05)$. Na tabela 2 , é apresentada, respectivamente, a prevalência com dois ou mais valores glicêmicos acima de 140, 160 e $180 \mathrm{mg} / \mathrm{dL}$, mas inferiores a $199 \mathrm{mg} / \mathrm{dL}$.

Tabela 1. Prevalência do diabetes e da hiperglicemia de estresse em 2.242 pacientes com eventos coronarianos agudos.

\begin{tabular}{lrrrrrrr}
\hline & \multicolumn{2}{c}{ Homens } & \multicolumn{2}{c}{ Mulheres } & \multicolumn{2}{c}{ Homens + mulheres } \\
& $\mathbf{n}$ & \% & $\mathbf{n}$ & \% & $\mathbf{n}$ & \% \\
Não-diabéticos & 1.019 & 62,8 & 375 & 58,6 & 1.394 & 61,6 \\
\hline Diabetes referido & 173 & 10.7 & 101 & 15,8 & 274 & 12,1 \\
Diabetes diagnosticado & 198 & 12,2 & 89 & 13,9 & 287 & 12,7 \\
Hiperglicemia de estresse & 232 & 14,3 & 75 & 11,7 & 307 & 13,6 \\
\hline Total & 1.622 & 100,0 & 640 & 100,0 & 2.262 & 100,0 \\
\hline
\end{tabular}

Diabéticos: duas glicemias casuais $>200 \mathrm{mg} / \mathrm{dL}$; hiperglicemia de estresse (duas ou mais glicemias casuais $\geq 140$ e $<200 \mathrm{mg} / \mathrm{dL}$ ).

Tabela 2. Prevalência de diabetes ou hiperglicemias de estresse durante o período de internação em 848 pacientes com eventos coronarianos agudos com diabetes não referido na admissão.

\begin{tabular}{lrrrrrrr} 
& \multicolumn{2}{c}{ Homens } & \multicolumn{3}{c}{ Mulheres } & \multicolumn{3}{c}{ Homens + mulheres } \\
Faixas glicêmicas (mg/dL) & $\mathbf{n}$ & $\%$ & $\mathbf{n}$ & $\%$ & $\mathbf{n}$ & $\%$ \\
140 a 159 & 80 & 13,3 & 25 & 58,6 & 105 & 12,8 \\
\hline 160 a 179 & 98 & 16,3 & 23 & 13,9 & 121 & 14,0 \\
180 a 199 & 54 & 9,0 & 27 & 15,8 & 81 & 9,4 \\
\hline > 200 & 371 & 61,4 & 190 & 11,7 & 561 & 64,39 \\
\hline Total & 603 & 100,0 & 265 & 100,0 & 868 & 100,0 \\
\hline
\end{tabular}


Nas figuras 1, 2 e 3 são apresentadas as prevalências de diabetes, hiperglicemia de estresse e indivíduos não-diabéticos com IAM em função das faixas etárias divididas em décadas. Em ambos os sexos foi observado gradual aumento relativo das prevalências de indivíduos com DM e com hiperglicemia de estresse com o aumento das respectivas faixas etárias até a sexta década $(\mathrm{p}<0,01)$, que tendeu a reduzir-se nas décadas seguintes. Nas mulheres, a prevalência de alterações glicêmicas na sexta década chega a representar quase metade $(48,11 \%)$ dos casos de IAM.

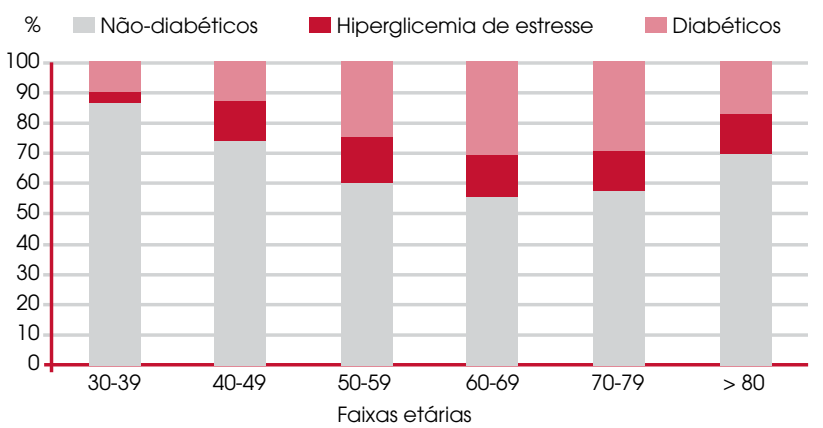

Figura 1. Prevalência de diabetes e glicemia de estresse em 2.446 pacientes com eventos coronarianos agudos, divididos por faixa etária, admitidos no InCor-SP.

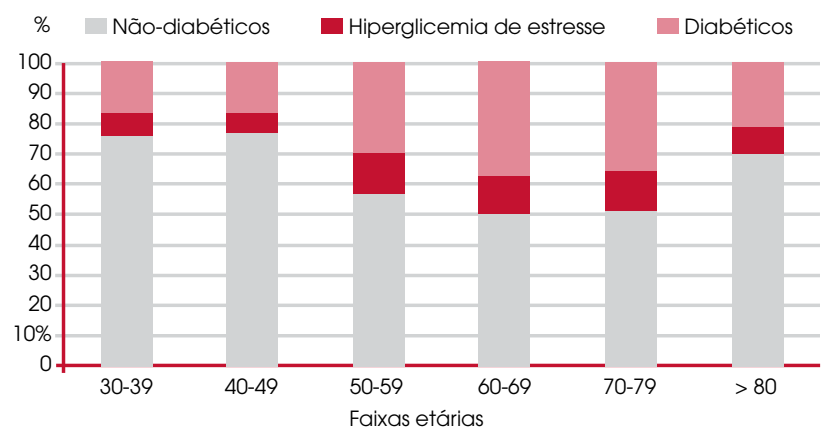

Figura 2. Prevalência de diabetes e hiperglicemia de estresse em 640 mulheres com eventos coronarianos agudos, divididas por faixa etária, admitidas no InCor-SP.

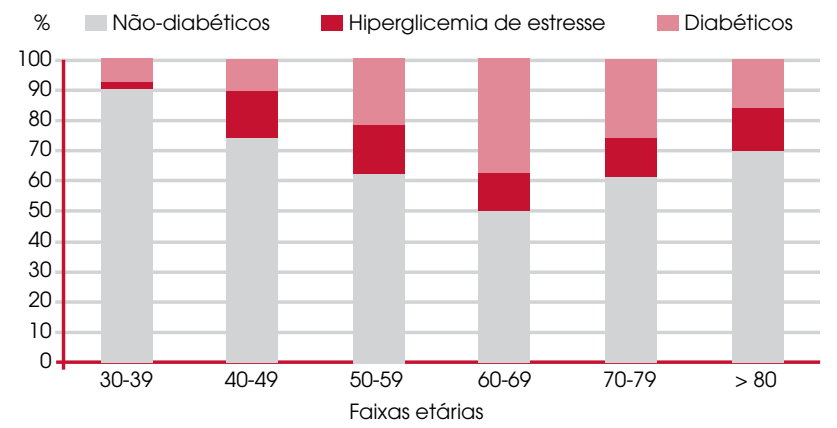

Figura 3. Prevalência de diabetes e hiperglicemia de estresse em 1.622 homens com eventos coronarianos agudos, divididos por faixa etária, admitidos no InCor-SP.
Quando se analisa os desfechos resultantes do evento miocárdico agudo durante o período de internação, observa-se uma porcentagem de óbitos significativamente maior nos homens diabéticos quando comparados aos não-diabéticos e com hiperglicemia de estresse $(\mathrm{p}<0,01)$. No sexo feminino, o número de óbitos não apresentou diferença estatisticamente significativa entre os grupos de mulheres não-diabéticas, com hiperglicemia de estresse e diabéticas (Figura 4). Quanto ao tipo de tratamento do IAM, o tratamento cirúrgico foi significativamente mais freqüente nos pacientes não-diabéticos em ambos os sexos quando comparados aos diabéticos e com hiperglicemia de estresse (homens: $\mathrm{p}<$ 0,001, mulheres p: <0,005). Nos pacientes submetidos ao tratamento cirúrgico, não foi observada diferença estatisticamente significativa de óbitos entre os grupos estudados (Figura 5).

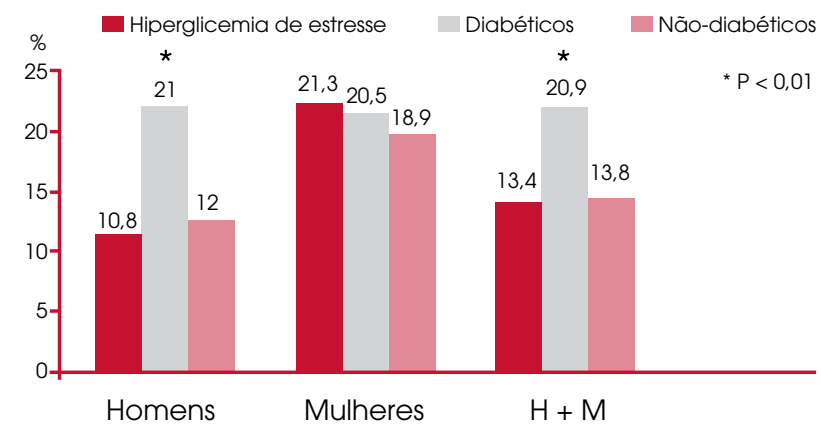

Figura 4. Prevalência de óbitos na internação de 2.262 eventos coronarianos agudos no InCor-SP.

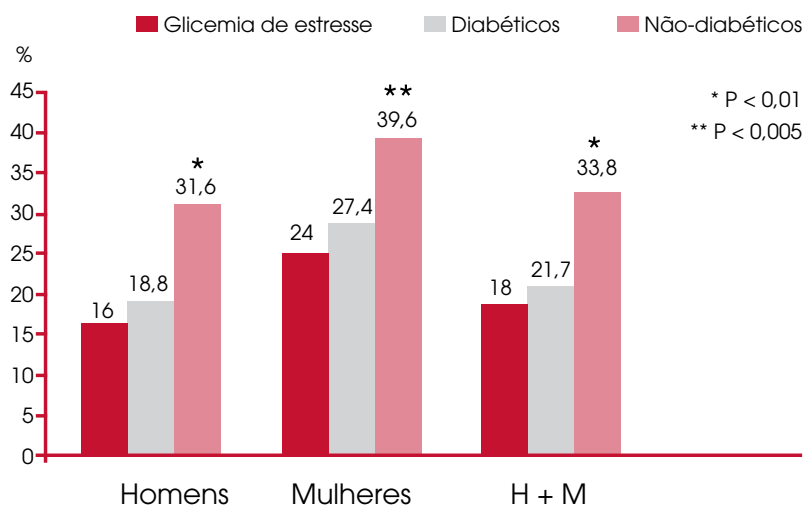

Figura 5. Porcentagem de tratamentos cirúrgicos na internação de 2.264 eventos coronarianos agudos no InCor-SP. 


\section{DISCUSSÃo}

Como conseqüência ao aumento da expectativa de vida da população e da melhor eficácia na prevenção e no tratamento das doenças infectocontagiosas, o papel das doenças crônicas, especialmente as doenças neoplásicas, metabólicas degenerativas e cardiovasculares, passam a ganhar especial importância como causa de mortalidade e morbidade. Em nosso país, entre as doenças crônicas, o diabetes e as doenças cardiovasculares atualmente estão ganhando crescente participação no contexto de problemas de saúde, especialmente nas camadas etárias mais idosas que tendem a aumentar $(26,27)$. Em contraposição, a característica da base alargada da pirâmide populacional constituída pela população jovem descrita nas décadas passadas, a distribuição populacional atual passa a adquirir a configuração de um cone (27). O diabetes, apesar de ser uma entidade nosológica freqüente, nem sempre é relatado como causa importante de mortalidade e motivo de internações hospitalares, uma vez que, usualmente, o diabetes não é referido como causa principal de admissão hospitalar ou de causa mortis, mas, sim, como causa secundária, especialmente quando associado aos eventos cardiovasculares agudos. Em pesquisa de causas de mortalidade, Lessa (9) demonstra que cerca de $50 \%$ dos pacientes com referência primária de doenças cardiovasculares nos atestados de óbito apresentavam como causa secundária ou terciária o diabetes melito. Além do mais, a detecção do diabetes como condição patológica prévia em pacientes com alterações cardiovasculares agudas é subestimada, uma vez que uma parcela elevada de pacientes com diabetes desconhece ser portadora dessa moléstia, fato este observado nos resultados do Censo Brasileiro de Prevalência do Diabetes realizado há aproximadamente uma década (28). Confirmando esses achados, observa-se no presente estudo um expressivo aumento da prevalência de diabetes entre os pacientes com IAM ao analisarmos as glicemias realizadas no período de internação. Nos 594 pacientes que não referiam diabetes, diagnosticamos DM em 307 (51,7\%) e hiperglicemia de estresse em $287(48,3 \%)$. Tanto nos homens como nas mulheres, os índices de prevalência dobraram após a inclusão dos resultados dos pacientes diagnosticados pela análise laboratorial.

Apesar de ser reconhecido como fator de risco para o desenvolvimento da doença coronariana, poucos são os estudos específicos, especialmente em nosso meio, que avaliam a prevalência de indivíduos diabéticos nos eventos coronarianos agudos. Para que pudéssemos comparar nossos resultados de prevalência do DM com IAM com os de outros autores, utilizamos os dados apresentados na caracterização de suas respectivas populações avaliadas em estudos que apresentavam objetivos primários diferentes do nosso. Em um estudo de metanálise visando a determinar os índices de mortalidade de pacientes diabéticos em síndromes coronarianas agudas, Donahue e cols. (29) observaram que dos 60.036 pacientes incluídos em 20 estudos com objetivos primários diversos, 10.613 eram diabéticos, o que correspondeu a 17,1\% dessa população. Rogers e cols. (30), objetivando avaliar as tendências temporais de tratamento de 1.514.292 de pacientes com IAM com base em banco de dados do Registro Nacional de Infarto do Miocárdio dos Estados Unidos, apresentaram prevalência de diabetes no período de 1990 a 1999 que variava entre $25,1 \%$ e $28,9 \%$ e Umpierrez e cols. (21), revendo prontuários de 1.886 pacientes com IAM, observaram a presença de hiperglicemia em $38 \%$ dos indivíduos, dos quais 26\% referiam diabetes antes da admissão e $12 \%$ não referiam história prévia de diabetes. Em estudos com coortes menores, Petursson e cols. (31), avaliando o estado glicometabólico imediato e 2,5 anos após o IAM, diagnosticaram diabetes em 99 (13\%) dos 762 indivíduos avaliados em um estudo feito em nosso País e Duarte e cols. (24), procurando avaliar a presença de marcadores inflamatórios metabólicos em 199 pacientes com síndrome isquêmica aguda, observaram prevalência de diabetes de $22,1 \%$. Portanto, a prevalência de $21,8 \%$ (12,1\% referido e $12,7 \%$ diagnosticado) de DM observado em nosso estudo não difere muito da maioria desses estudos, entretanto não pudemos comparar a proporção de diabetes referido ou diagnosticado uma vez que somente um estudo apresentou essa característica (21).

Quanto à prevalência da hiperglicemia de estresse, os dados disponíveis demonstram grande variabilidade de resultados em virtude de diferentes critérios de sua definição diagnóstica, especialmente com referência a seus limiares glicêmicos inferiores. As respectivas prevalências observadas da hiperglicemia de estresse em pacientes com síndromes coronarianas agudas foram: $49 \%$ dos 2.127 pacientes (glicemias entre 130 e $180 \mathrm{mg} / \mathrm{dL}$ ) no estudo de Foo e cols. (20), 38\% dos 106 pacientes (glicemias entre 126 e $200 \mathrm{mg} / \mathrm{dL}$ ) no estudo de Nordin e cols. (18) e $12 \%$ dos 2.030 pacientes (glicemias entre 126 e $200 \mathrm{mg} / \mathrm{dL}$ ) no estudo de Umpierrez e cols. (21). 
Em trabalho de metanálise, Capes e cols. (16), selecionando oito estudos de pacientes com IAM sem referência de diabetes com casuísticas variadas e diferentes critérios de diagnóstico para a hiperglicemia de estresse apresentaram prevalências que variaram entre 3\% e 71\%.

A presença da hiperglicemia estresse, observada em $15,4 \%$ de nossos pacientes que não referiam diabetes, indicaria que estes quando submetidos ao estresse associado ao evento agudo coronariano apresentam menor capacidade secretória de insulina que caracterizaria a fase préclínica do diabetes, geralmente assintomática, mas de elevado risco para o desenvolvimento da doença coronariana. Essa condição foi evidenciada por Norhammar e cols. (23), que pela realização de um teste de tolerância oral a glicose três meses após a internação de 181 pacientes com IAM que não referiam diabetes, diagnosticaram diabetes e intolerância à glicose ( 2 horas) em, respectivamente, $36 \%$ e $40 \%$ desses pacientes. Avaliando 168 pacientes classificados como não-diabéticos na admissão hospitalar com IAM, Wallander e cols. (32) demonstraram deterioração da função da célula-beta pancreática avaliada pelo o índice insulinogênico (IGI), aumento da resistência à insulina determinada pelo HOMA-IR e piora da tolerância à glicose determinada pelo teste de tolerância à glicose realizado na alta de cada um desses indivíduos. Com base em seus achados, concluem que a hiperglicemia de estresse refletiria um estado de disfunção da regulação glicêmica que precede o IAM. Esses dados confirmam os resultados de estudos prospectivos que demonstraram que o risco cardiovascular de pacientes sem diabetes clínico, mas com disglicemia e intolerância à glicose, é semelhante ou até mesmo maior do que os de pacientes com glicemias de jejum alteradas (13-15).

A demonstração de que a hiperglicemia causal ou a pós-prandial apresenta o mesmo ou até um maior risco cardiovascular observado no diabetes clínico sugere que as alterações endoteliais observadas nesses pacientes dependeriam não somente da ação direta da hiperglicemia, mas também da presença de outros fatores de risco componentes da síndrome metabólica (SM) que é uma condição presente na maioria dos pacientes com diabetes tipo 2 (33). Como usualmente os pacientes com diabetes tipo 2 apresentam em toda sua história natural da doença a SM e a resistência à insulina, seu principal componente, o risco de apresentar um evento cardiovascular agudo é também elevado em pacientes que ainda não apresentam o diabetes em sua forma manifesta, mas também nas condições de glicemia de jejum alterada e de hiperglicemia pós-prandial $(13,14)$.
O impacto da hiperglicemia de estresse como fator de piores desfechos tem sido estudado por diferentes estudos que demonstraram significante aumento da mortalidade hospitalar e após a alta de pacientes com eventos cardiovasculares agudos, entretanto não se conseguiu estabelecer um valor glicêmico mínimo que condicionaria esse risco $(16,20)$. Confirmando dados de outros estudos, observamos tanto nos pacientes diabéticos quanto nos com hiperglicemia de estresse, maior freqüência de óbitos durante o período de hospitalização pós-infarto (16-22). Uma evidência indireta que confirma a ação deletéria da hiperglicemia de estresse no período admissional nos desfechos do IAM foi evidenciada por Malmberg e cols. (34) no estudo Digami que, pela correção do controle glicêmico obtido pela introdução precoce da insulinização intensiva no período intra-hospitalar obteve significativa redução da mortalidade desses pacientes tanto na alta como após um ano de seguimento ambulatorial. $\mathrm{O}$ maior risco de mortalidade de pacientes diabéticos com IAM foi também evidenciado por outros estudos que também demonstraram a maior gravidade do grau de lesão miocárdica, o aumento de complicações cardiológicas e maior número de artérias comprometidas quando comparados a indivíduos não-diabéticos (35-38).

Quando analisamos nossa população de estudo em relação às faixas etárias, observamos que enquanto o número de indivíduos não-diabéticos com infarto cresce gradualmente a partir da quarta década de vida atingindo sua maior prevalência em torno da sétima década, os pacientes com diabetes e com hiperglicemia de estresse apresentam sua maior prevalência na sexta década de vida, sugerindo que a presença do diabetes não somente aumenta, como também antecipa para uma fase etária mais precoce o risco de desenvolver um evento cardiovascular agudo. Constatamos que entre os $60 \mathrm{e}$ os 69 anos a prevalência de indivíduos com DM e com HE somada chega a corresponder a $43,89 \%$ dos indivíduos dessa faixa etária, valor este mais expressivo no sexo feminino, que correspondeu a praticamente metade $(48 \%)$ das mulheres com IM nessa faixa de idade (Figura 2). Não temos uma explicação satisfatória para esse aumento da prevalência em mulheres, que talvez poderia estar associado a menor proteção hormonal associada ao desenvolvimento da menopausa. A maior precocidade do aparecimento do diabetes em nossos pacientes pode ser atribuída à presença do diabetes como fator de risco cardiovascular adicional ao risco natural associado ao envelhecimento. 
Quanto ao tipo de tratamento utilizado, observamos em pacientes de ambos os sexos de nosso grupo de estudo menor freqüência de terapêutica cirúrgica nos pacientes diabéticos e com hiperglicemia de estresse, quando comparados aos indivíduos não-diabéticos. A menor freqüência de intervenções cirúrgicas pode ser explicada pela maior gravidade da função cardíaca nos pacientes diabéticos e pelo grau de comprometimento arterial que freqüentemente é difuso, podendo ser um fator de sua contra-indicação $(36,38)$. Apesar de não haver evidências conclusivas de que pacientes diabéticos apresentem infartos mais extensos, é mais comum a presença de insuficiência cardíaca e choque cardiogênico nesses pacientes em função de uma disfunção diastólica preexistente $(36,39)$. Outra explicação se refere à maior cautela de indicação cirúrgica, considerando serem piores os desfechos pós-intervenção, especialmente por angioplastia (40). Estudos mais recentes indicam que mesmo com o uso de stents revestidos, a evolução dos pacientes diabéticos foi mais desfavorável que nos indivíduos não-diabéticos (41). Em nossa população de estudo não observamos diferenças significativas em relação à mortalidade durante o período de internação no grupo de diabéticos e com hiperglicemia de estresse quando comparado aos pacientes não-diabéticos.

Em conclusão, nosso estudo demonstrou que a prevalência das alterações glicêmicas em pacientes que apresentam o IAM é mais elevada do que as referidas em quadros estatísticos por não incluírem as alterações pré-clínicas do diabetes que apresentam igual elevado risco cardiovascular. Como recentemente é observado nítido aumento da prevalência do diabetes e crescente contribuição das alterações glicêmicas clínicas e subclínicas como fator para o desenvolvimento e a piora dos desfechos das síndromes coronarianas agudas (11), é fundamental que seja enfatizada maior atenção para o diagnóstico e o tratamento precoce do diabetes, assim como de seus fatores condicionantes, como a obesidade e o sedentarismo.

\section{REFERÊNCIAS}

1. King $\mathrm{H}$, Albert RE, Herman WH. Global burden of diabetes 1995-2025: prevalence, numerical estimates, and projections. Diabetes Care. 1998;21:1414-31.

2. Boyle JP, Honeycutt AA, Narayan KM, Hoerger TJ, Geiss LS, $\mathrm{Chen} \mathrm{H}$ et al. Projection of diabetes burden through 2050: impact of changing demography and disease prevalence in the US. Diabetes Care. 2001;24:1936-40.
3. Ministério da Saúde do Brasil e Organização Panamericana da Saúde (OPAS). Avaliação do Plano de Reorganização da Hipertensão Arterial e o Diabetes Mellitus no Brasil 2004; 23-47.

4. Morrish NJ, Wang SL, Stevens LK, Fuller JH, Keen H. Mortality and causes of death in the WHO Multinational Study of Vascular Disease in Diabetes. Diabetologia. 2001;44 (Suppl 2):S14-S21.

5. Alexander CM, Landsman PB, Teutsch SM. Diabetes mellitus, impaired fasting glucose, atheroclerotic risk factors, prevalence and coronary hearth disease. Am J Cardiol. 2000;86:897-902.

6. Beckman JA, Creager MA, Libby P. Diabetes and atherosclerosis: epidemiology, pathophysiology, and management. JAMA. 2002;287:2570-81.

7. Meigs JB. Epidemiology of cardiovascular complications in type 2 diabetes. Acta Diabetol. 2003;40:S358-61.

8. Kannel WB, McGee DL. Diabetes and cardiovascular disease: the Framingham Study. JAMA. 1999;241:2035-8.

9. Lessa I. Relative mortality trends for diabetes mellitus in the Brazilian state capitals (1950-1985). Bol Oficina Sanit Panam. 1992;113(3):212-7

10. Balkau B, Rschwege E. Insulin resistance: an independent risk factor for cardiovascular disease? Diabetes Obes Metab. 1999;Suppl1:S23-31.

11. Gu K, Connie CC, Harris MI. Diabetes and decline in hearth disease mortality in US adults. JAMA. 1999;281:1291.

12. American Diabetes Association, Clinical Practice Recommendations: 2006 Diagnosis and Classification of Diabetes mellitus. Diabetes Care. 2006;29:S43-48.

13. Meigs JB, Nathan DM, D'Agostino RB Sr. Wilson PW Fasting and post-challenge glycemia and cardiovascular disease risk: the Framingham Off-Spring Study. Diabetes Care. 2002;25:1845-50.

14. DECODE Study Group Glucose tolerance and cardiovascular mortality: comparison of fasting and 2-hour diagnostic criteria. Arch Intern Med. 2001;161:397-405.

15. Hu FB, Stamper MJ, Haffner SM, Solomon CG, Willett WC, Manson JE. Elevated risk of cardiovascular disease of type 2 diabetes. Diabetes Care. 2002;25:1129-34.

16. Capes SE, Hunt D, Malmberg K, Gerstein HC. Stress hyperglycaemia and increased risk of death after myocardial infarction in patients with and without diabetes: a systematic overview. Lancet. 2000;355:773-8.

17. Oswald G, Smith C, Betteridge J, Yudkin J. Determinants and importance of stress hyperglycemia in non-diabetic patients with myocardial infarction. Br Med J. 1986;293:917-22.

18. Nordin C, Amirrudin R, Ricker L, Choi J, Marantz PR. Diabetes and stress hyperglycemia with myocardial infarctions and an urban municipal hospital: prevalence and effect on mortality Cardiol Rev. 2005;13:223-30.

19. Norhammar AM, Rydèn L, Malmberg K. Admission Plasma Glucose - Independent risk factor for long-term prognosis after myocardial infarction even in non-diabetic patients. Diabetes Care. 1999;22:1827-31.

20. Foo K, Cooper J, Deaner A, Knigh C, Suliman A, Ranjadoyalan $\mathrm{K}$, et al. A single serum glucose measurement predicts adverse outcomes across the whole range of acute coronary syndrome. Heart. 2003;89:512-6.

21. Umpierrez GE, Isaacs SD, Bazargan N, You X, Thaler LM, Kitabchi AE. Hyperglycemia: A independent marker of in-hospital mortality in patients with undiagnosed diabetes. J Clin Endocrinol Metab. 2002;87:978-82. 
22. Coutinho M, Gerstein HC, Wang Y, Yusuf S. The relationship between glucose and incident cardiovascular events. Diabetes Care. 1999;22:233-40.

23. Northammar A, Tenerz A, Nilsson G. Glucose metabolism in patients with acute myocardial infarction and no previous diagnosis of diabetes mellitus: a prospective study. Lancet. 2002;359:2140-4.

24. Duarte ER, Pellanda LC, Portal VL. Inflammatory, lipid, and metabolic profile in acute ischemic syndrome. Correlation with hospital and post-hospital events. Arq Bras Cardiol. 2005;84:122-9.

25. World Health organization Definition, diagnosis and classification of diabetes mellitus and its complications. Report of a WHO consultation. Part 1 Diagnosis and classification of diabetes mellitus 1999 .

26. Instituto Brasileiro de Geografia e Estatística IBGE. Estatísticas de indicadores sociais. Disponível em: www.ibge/pop.gov.br. (Acesso em 20 dez 2006).

27. Fundação Estadual de Análise de Dados - Seade. Disponível em: www.seade.gov.br. (Acesso em 20 dez 2006).

28. Ministério da Saúde do Brasil. Censo nacional sobre a prevalência de diabetes no Brasil 1989. Disponível em: www.diabetes.org.br (Acesso em 20 dez 2006).

29. Donahue SM, Steward GC, McCabe CH, Mohanavelu S, Murphy SA, Cannon CP et al. Diabetes and mortality following acute coronary syndromes. JAMA. 2007;298:765-75.

30. Rogers WJ, Canto JG, Lambrew CT, Tiefenbrunn AJ, Kinkaid B, Shoultz DA et al. Temporal trends in the treatment of over 1,5 million patients with myocardial infarction in U.S. from 1990 through 1999. J Am Coll Cardiol. 2000;36:2056-5063.

31. Petursson $P$, Herlitz J Caldahl K, From-Attebring $M$, Sjoland $H$, Gudbjornsdottir $S$ et al. Association between glycometabolic status in the acute phase and $2 \frac{1}{2}$ years after an acute coronary syndrome. Scand Cardiovasc J. 2006;40:45-51.

32. Wallander M, Bartnick M, Efendic S, Hamsten A, Malmberg $O$, Ohrvik J et al. A Beta cell dysfunction in patients with acute myocardial infarction but without previously known type 2 diabetes: a report from the Gami study. Diabetologia. 2005;45:2229-35.

33. Alexander CM, Landsman PB, Teutsch SM, Haffner SM. NCEPdefined metabolic syndrome, diabetes. and prevalence of coronary hearth disease among NHANES III participants age 50 and older. Diabetes. 2003;52:1210-14.
34. Malmberg K, Ryden L, Efendic S, Herlitz J, Niol P, Walstrom A et al. Randomized trial of insulin glucose infusion followed by subcutaneous insulin treatment in diabetic patients with acute myocardial infarction (Digami Study ): effects on mortality at 1 year. J Am Coll Cardiol. 1995:26:57-65.

35. Aronson D, Rayfeld EJ, Chesebro JH. Mechanisms determining and outcome of diabetic patients who had an acute myocardial infarction. Ann Intern Med. 1997;126:296-306.

36. Lehto S, Pyorala K, Metiennen M. Myocardial infarct size and mortality in patients with non-insulin dependent diabetes mellitus. J Intern Med. 1994;236:291-7.

37. Orlander PR, Goff DC, Morrissey M, Ramsey DJ, Wear ML, Labarthe DR et al. The relation of diabetes to the severity of acute myocardial infarction and post-miocardial infarction survival in Mexican-American and Non-Hispanic whites The Corpus Christi Hearth Project. Diabetes. 1994;43:897-902.

38. Stone PH, Muller JE, Hartwell T, York BJ, Rutherford JD, Parker CB et al. The effect of diabetes mellitus on the prognosis and serial ventricular function after acute myocardial infarction: contribution of both coronary disease and diastolic ventricular dysfunction to adverse prognosis The Milis Study Group. J Am Coll Cardiol. 1989;14:49-57.

39. Fava S, Azzopardi j, Muscat HA, Fenech FF. Factors that influence outcome in diabetic subjects with myocardial infarction. Diabetes Care. 1993;16:1615-8.

40. Sobel BE, Frye R, Dedre KM. Burgeoning dilemmas in the management of diabetes and cardiovascular disease. Rationale for bypass angioplasty revascularization investigation 2 diabetes (Bari D2). Trial Circulation. 2003;107:636-42.

41. Stettler C, Allernann S, Egger M, Windecker S, Meier B, Diern P. Efficacy of drug eluting stents in patients with and without diabetes mellitus: indirect comparisons of controlled. Heart. 2006; 92:650-7.

\section{Endereço para correspondência:}

Antonio Carlos Lerario

Rua Itapeva, 378, cj. 133

01332-901 São Paulo, SP

E-mail: alerario@terra.com.bre aclerario@terra.com.br 